\title{
Electrokinetic effect of the Loma Prieta earthquake calculated by an entire-Earth FDTD solution of Maxwell's equations
}

\author{
Jamesina J. Simpson and Allen Taflove \\ Department of Electrical and Computer Engineering, Northwestern University, Illinois, USA \\ Received 2 February 2005; revised 31 March 2005; accepted 6 April 2005; published 10 May 2005.
}

[1] We report what we believe to be the first threedimensional computational solution of the full-vector Maxwell's equations for hypothesized pre-seismic electromagnetic phenomena propagated within the entire Earth-ionosphere cavity. Periodic boundary conditions are used in conjunction with a variable-cell finite-difference time-domain (FDTD) space lattice wrapping around the complete Earth-sphere and extending $\pm 100 \mathrm{~km}$ radially from sea level. This technique permits a direct timedomain calculation of round-the-world ULF/ELF propagation accounting for arbitrary horizontal as well as vertical geometrical and electrical inhomogeneities/ anisotropies of the excitation, ionosphere, lithosphere, and oceans. In this study, we model electrokinetic currents at depths of $2.5 \mathrm{~km}$ and $17 \mathrm{~km}$ near the hypocenter of the Loma Prieta earthquake and compare the FDTDcalculated surface magnetic field to analytical results and measurements previously reported in the literature. We accommodate the complete physics introduced by impulsive electromagnetic wave propagation through the conductive Earth, and hence illustrate the importance of solving the full Maxwell's equations when modeling current sources within the Earth's crust. Our calculated spectra agree qualitatively with those reported by FraserSmith et al. (1990). Citation: Simpson, J. J., and A. Taflove (2005), Electrokinetic effect of the Loma Prieta earthquake calculated by an entire-Earth FDTD solution of Maxwell's equations, Geophys. Res. Lett., 32, L09302, doi:10.1029/ 2005 GL022601.

\section{Introduction}

[2] Anomalous ultra low frequency (ULF: 0-3 Hz) and extremely low-frequency (ELF: $3 \mathrm{~Hz}-300 \mathrm{~Hz}$ ) electromagnetic (EM) wave phenomena occurring prior to major earthquakes have been of particular interest for many years [Park et al., 1993; Johnston, 1997]. A number of physical mechanisms related to hypothetical earthquake precursors have been proposed to explain the origin of such observations [Park et al., 1993]. The existence and nature of any EM signatures associated with these mechanisms currently remains unproven and unclear, however. For example, analysis of potential signatures were presented by Molchanov et al. [1995] and Kulchitsky et al. [2004]; however, Molchanov et al. [1995] neglected wave propagation, and Kulchitsky et al. [2004] provided a scalar two-dimensional analysis which neglected the Earth's curvature. Our overall goal for the novel EM modeling

Copyright 2005 by the American Geophysical Union. 0094-8276/05/2005GL022601\$05.00 work described here is to provide a more rigorous analysis of this issue than previously published, thereby placing hypothetical pre-seismic EM phenomena on a firmer physical basis.

[3] This letter reports what we believe to be the first three-dimensional (3-D) computational solution of the full-vector Maxwell's equations for hypothesized preseismic EM phenomena within the entirety of the Earth-ionosphere. Applying the finite-difference time-domain (FDTD) method [Taflove and Hagness, 2000], we obtain a direct time-domain calculation of round-theworld ULF/ELF propagation accounting for arbitrary horizontal as well as vertical geometrical and electrical inhomogeneities/anisotropies of the excitation, ionosphere, lithosphere, and oceans. Using this rigorous numerical model, we accommodate the complete physics introduced by impulsive electromagnetic wave propagation through the conductive Earth and subsequent reverberation through the entire Earth-ionosphere waveguide, and hence illustrate the importance of solving the full Maxwell's equations when modeling current sources within the Earth's crust.

[4] Previously, we reported applying FDTD to efficiently model the complete Earth-sphere at ELF using a 3-D, spherical-coordinate, latitude-longitude space lattice with periodic boundary conditions [Simpson and Taflove, 2002a, 2002b, 2003, 2004a]; see http://www.ece.northwestern.edu/ ecefaculty/taflove/3Dmovietext@gif.avi. To minimize the effects of increasing space-cell eccentricity upon approaching a pole, we employed an adaptive cell-combination technique applied to adjacent grid cells in the east-west direction, thereby allowing maintenance of the time-step at nearly the level permitted by the Courant stability condition for the square equatorial cells. This technique permits a standard laboratory computer with 2 GB of random access memory to generate high-resolution $(\sim 40 \times 40 \times 5 \mathrm{~km})$ modeling results for global, fully 3-D, impulsive ULF/ELF propagation within the entire Earth-ionosphere cavity to within $\pm 100 \mathrm{~km}$ of sea level.

[5] In this study, we apply our entire-Earth FDTD numerical model to calculate EM emissions generated by electrokinetic currents located near the hypocenter of the Loma Prieta earthquake. Such currents have been proposed to explain one of the primary examples of hypothetical precursory EM phenomena to date: the anomalous magnetic fields reported by Fraser-Smith et al. [1990] prior to the Loma Prieta earthquake. We compare the FDTD surface magnetic field calculations to analytical results and measurements previously reported in the literature, and find that our calculated 


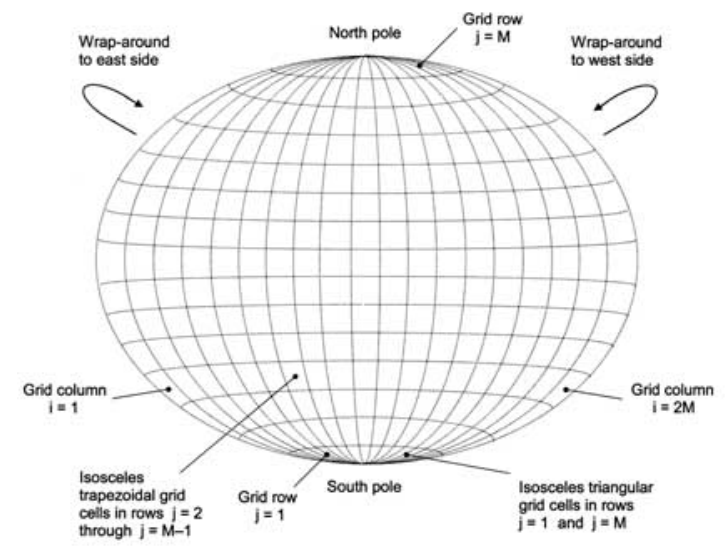

Figure 1. General layout of the of the 3-D FDTD Maxwell's equations space-lattice covering the complete Earth-sphere as seen from a TM plane at a constant radial coordinate.

spectra agree qualitatively with those reported by FraserSmith et al. [1990].

\section{The 3-D Latitude-Longitude FDTD Grid}

[6] Figure 1 illustrates the general layout of the FDTD space-lattice as seen from the transverse magnetic (TM) plane at a constant radial coordinate [Simpson and Taflove, 2004a]. The lattice is a logically Cartesian $2 M \times M \times K$ cell arrangement, where $M$ is a power of 2 . We see that the grid cells follow lines of constant latitude, $\theta=$ constant, where $\theta$ is the usual spherical angle measured from the north pole; and along lines of constant longitude, $\phi=$ constant, where $\phi$ is the usual spherical azimuthal angle measured from a specified prime meridian. In this manner, each TM plane of the grid shown in Figure 1 is comprised of isosceles trapezoidal cells away from the north and south poles [Simpson and Taflove, 2004a], and isosceles triangular cells at the poles [Simpson and Taflove, 2004a]. Similarly, each transverse electric (TE) plane at a constant radial coordinate is comprised of isosceles trapezoidal cells away from the north and south poles, and a polygon cell at each pole [Simpson and Taflove, 2004a].

[7] We choose to have the same angular increment in latitude, $\Delta \theta=\pi / M$, for each cell in the grid. Thus, the south-north span of each trapezoidal or triangular grid cell is $\Delta_{\mathrm{s}-\mathrm{n}}=\pi R / M$, where $R$ is the radial distance from the center of the Earth. To maintain square or nearly square grid cells near the equator, we select the baseline value of the angular increment in longitude, $\Delta \phi$, to equal $\Delta \theta$. However, this causes the west-east span of each cell, $\Delta_{\mathrm{w}-\mathrm{e}}=R \Delta \phi \sin \theta$, to be a function of $\theta$. This could be troublesome for cells near the north and south poles where $\theta \rightarrow 0$ and $\theta \rightarrow \pi$, respectively. There, the geometrical eccentricity of each cell, $\Delta_{\mathrm{s}-\mathrm{n}} / \Delta_{\mathrm{w}-\mathrm{e}}=\Delta \theta /(\Delta \phi \sin \theta)$, would become quite large, and the numerical stability and efficiency of the FDTD algorithm would be degraded. We mitigate this problem by merging pairs of adjacent cells of the TM plane in the west-east direction, effectively halving the cell eccentricity [Simpson and Taflove, 2004a]. This process can be repeated several times as the grid approaches a pole, allowing the user to specify a maximum allowable cell eccentricity.

[8] The wrap-around or joining of the east and west edges of the lattice occurs along a specific line of constant longitude, or meridian. This joining is, in effect, a periodic boundary condition applied at each $j$-row of lattice cells, whether trapezoids or triangles [Simpson and Taflove, 2004a].

[9] Given the above assumptions, Ampere's Law in integral form can be applied to develop an FDTD timestepping relation for each electric $E$ field components of the grid [Simpson and Taflove, 2004a]. Similarly, Faraday's Law in integral form can be applied to develop an FDTD time-stepping relation for the magnetic $H$ field components of the grid [Simpson and Taflove, 2004a].

\section{Details of the Electrokinetic Study}

[10] In our present study, we focus on modeling EM emissions resulting from proposed electrokinetic currents in the Earth's crust near the hypocenter of the Loma Prieta earthquake along the San Andreas Fault. These current sources are believed to arise when fluid flows through a porous medium in the presence of an electrical double layer at a solid-liquid interface [Park et al., 1993]. Fenoglio et al. [1995] previously modeled electrokinetic currents using a Biot-Savart model to explain the behavior of the anomalous precursory EM data reported by Fraser-Smith et al. [1990] prior to the Loma Prieta earthquake. For their study, they used a layered half-space geometry to characterize the surface magnetic fields directly above the electrokinetic currents. Subsequently, Majaeva et al. [1997] modeled electrokinetic currents using a layered cylindrical model that also accounted for diffusion.

[11] In the present study, we report a significantly more rigorous calculation of electrokinetic EM emissions relative to those of Fenoglio et al. [1995] and Majaeva et al. [1997]. Using the entire-Earth 3-D FDTD grid briefly described above, we solve the full-vector Maxwell's equations to account for the complete physics introduced by impulsive wave propagation through a conductive Earth and subsequent round-the-world propagation, which has been shown to be an important factor at ULF/ELF [Simpson and Taflove, 2004a]. Our model utilizes topographic and bathymetric data from the NOAA-NGDC "Global Relief CD-ROM". These data are mapped onto our 3-D space lattice with an assumed resolution of $40 \times 40 \times 5 \mathrm{~km}$ at the equator. For the lithosphere, conductivity values are assigned according to Hermance [1995], depending upon the location of the E component (i.e., below an ocean or within a continent). For the atmosphere, both day- and nighttime exponential conductivity profiles used by Bannister [1985] are assumed, with early evening (the timing of the Loma Prieta earthquake) occurring along the San Andreas Fault.

[12] Fenoglio et al. [1995] modeled a current associated with fluid flow into a $200 \mathrm{~m}$ by $1000 \mathrm{~m}$ shear fracture at a depth of $17 \mathrm{~km}$. According to their model, repeated pulses are generated by successive $25-\mathrm{m}$ fractures along the $200 \mathrm{~m}$ long compartment. In the present study, we first simulate one such pulse occurring along a 17-km-deep horizontal electric field at $121.88^{\circ} \mathrm{W}, 37.04^{\circ} \mathrm{N}$, near the hypocenter of the Loma Prieta earthquake. This is essentially the space- 


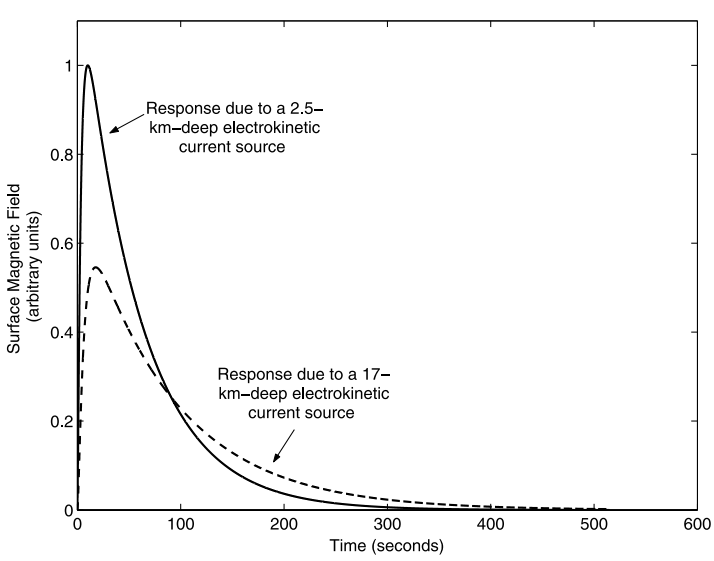

Figure 2. Comparison of the FDTD-calculated time waveforms for the horizontal surface $H$-field component resulting from one electrokinetic current source: (1) 17-kmdeep; and (2) 2.5-km-deep. See color version of this figure in the HTML.

time Green's function of the global Earth-ionosphere waveguide as excited at the hypocenter. To ensure a smooth onset of the excitation, the current is assumed to linearly increase to its maximum value at $1000 \Delta t$, where $\Delta t=3.0 \mu \mathrm{s}$.

[13] Our present grid resolution at $40 \times 40 \times 5 \mathrm{~km}$ requires us to model the $25-\mathrm{m}$ current source onto a $40-\mathrm{km}$ long electric field component. This does not, however, upset our calculated surface magnetic field values after normalization. In fact, we can determine the resulting surface magnetic field due to successive $25-\mathrm{m}$ fractures along the 200 m-long compartment by modeling multiple current signals on the same buried horizontal electric field component. This is due to the alignment of the current sources along the same general direction, allowing each to contribute linearly in the far-field to the overall dipole moment. The total surface magnetic field due to any number of cascading, parallel pulses occurring within the span of one grid-cell component is therefore simply the superposition of multiple time-delayed versions of the response caused by a single electrokinetic current source.

\section{Discussion of Results}

[14] We now report the results of our full-vector, 3-D FDTD Maxwell's equations model. Figure 2 shows the time-waveforms of the horizontal surface $H$-field component generated by a single electrokinetic pulse at a depth of $2.5 \mathrm{~km}$ and $17 \mathrm{~km}$. Using the Lemieux parallel processing supercomputer at the Pittsburgh Supercomputing Center, we calculated the initial 25 seconds using our FDTD model, and thereafter applied Prony's method [Academic Technologies, 2002] to extend the tail of the response to its ultimate decay. The time waveform generated by the $17-\mathrm{km}$-deep source is subsequently used as an equivalent Green's function in the discussion below. Interestingly, we see that the late-time response of the deeper source is higher due to the longer diffusion time within the lossy crustal medium.

[15] Figure 3 compares the normalized time waveform of the response of the horizontal surface $H$-field component due to eight successive 17-km-deep electrokinetic current pulses as reported by Fenoglio et al. [1995] (calculated

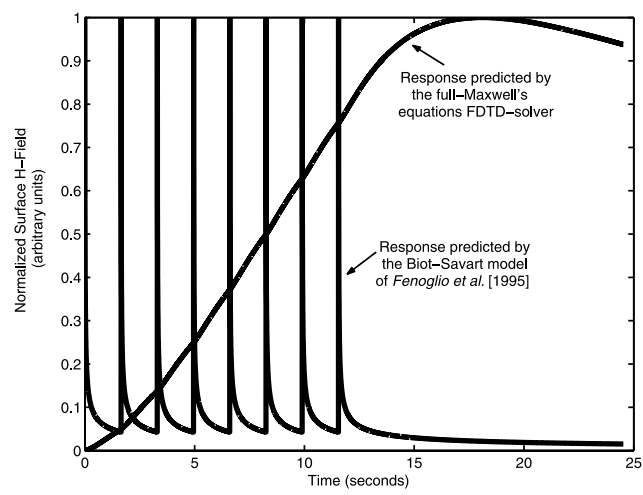

Figure 3. Comparison of the time waveforms for the horizontal surface magnetic field component due to $17-\mathrm{km}$ deep electrokinetic currents associated with eight successive 25-m fractures occurring 1.65 seconds apart along a $200 \mathrm{~m}$ long compartment: (1) as predicted by the Biot-Savart model of Fenoglio et al. [1995]; and (2) as predicted by the 3 -D FDTD Maxwell's equations model. See color version of this figure in the HTML.

using a simple Biot-Savart model), with the predictions of our FDTD Maxwell's equations model. For both sets of results, each current source pulse occurs after a time delay of about 1.65 seconds. As shown in Figure 3, the Maxwell's equations model yields a vastly different temporal response. We believe that this difference has two primary and interlinked causes: (i) The FDTD Maxwell's equations model properly accounts for temporal integration due to EM diffusion through $17 \mathrm{~km}$ of lithosphere; (ii) The FDTD Maxwell's equations model properly accounts for reverberation within the Earth-ionosphere waveguide, which has low propagation loss below $0.2 \mathrm{~dB} / \mathrm{Mm}$ for frequencies below $10 \mathrm{~Hz}$ [Bannister, 1984]. In this manner, the instantaneous response of the surface $H$-field is properly

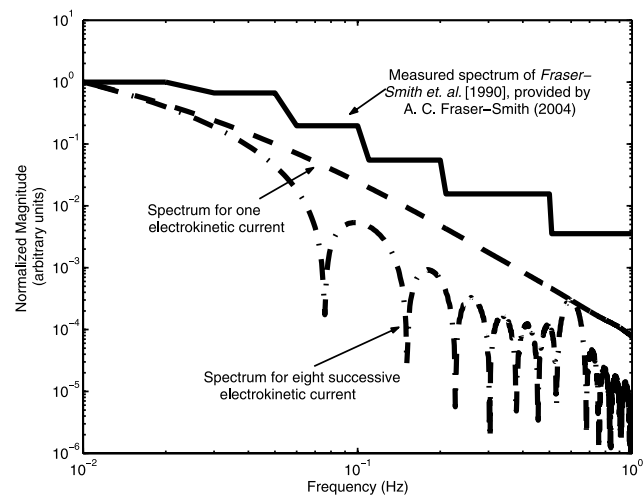

Figure 4. Comparison of the spectra for the horizontal surface $H$-field component: (1) measured by Fraser-Smith et al. [1990] and A. C. Fraser-Smith (personal communication, 2004); (2) FDTD result for eight electrokinetic currents associated with successive $25-\mathrm{m}$ fractures occurring 1.65 seconds apart (corresponding to the comparatively large spectral peak at $0.61 \mathrm{~Hz}$ ) along a 200 m-long compartment and at a depth of 17-km-deep; and (3) FDTD result for one electrokinetic current at $17-\mathrm{km}$ depth. See color version of this figure in the HTML. 
influenced by current sources occurring much earlier in time.

[16] Figure 4 compares $H$-field frequency spectra from $0.01-1.0 \mathrm{~Hz}$ for three sets of data: (i) measurements reported by Fraser-Smith et al. [1990] and A. C. FraserSmith (personal communication, 2004); (ii) FDTD calculation for one electrokinetic current pulse located at a depth of $17 \mathrm{~km}$, as shown in Figure 2; and (iii) FDTD calculation for eight successive electrokinetic current pulses (at intervals of 1.65 seconds) located at a depth of $17 \mathrm{~km}$. All three spectra are normalized at $0.01 \mathrm{~Hz}$ to illustrate their relative rates of decay with increasing frequency, and are graphed on a common logarithmic frequency and amplitude scale. There is qualitative agreement of the measured and calculated results. Assuming that the hypothesized electrokinetic current actually underlies the Fraser-Smith et al.observations, the deviations between the FDTD-calculated spectra and the measurements have two probable causes: (i) assumption of a lithospheric conductivity in the FDTD model that exceeds the actual value; and (ii) a measurement artifact reported by Fraser-Smith et al. [1990] wherein instrumentation saturation likely occurred at the lowest observed frequencies. In the first case, an excessive lithospheric conductivity would cause the FDTD-calculated spectra at higher frequencies to be unduly attenuated, thereby bending the calculated curves downward below the Fraser-Smith et al. data. In the second case, the Fraser-Smith et al. data point at $0.01 \mathrm{~Hz}$ would have too small a value, thereby unduly flattening their measured spectra.

\section{Conclusions and Ongoing Work}

[17] We have presented what we believe to be the first rigorous 3-D computational solution of the full-vector Maxwell's equations for hypothesized pre-seismic EM phenomena propagated within the entire Earth-ionosphere cavity. Using the robust FDTD method, we simulated electrokinetic currents near the hypocenter of the Loma Prieta earthquake and compared the calculated surface $H$-field with analytical results and measurements previously reported in the literature. Our entire-Earth FDTD grid allowed us to properly model complex, interlinked phenomena due to EM diffusion through the lithosphere and EM wave reverberation due to round-the-world propagation within the Earth-ionosphere cavity. We found that the FDTD-calculated spectra for the surface $H$-field qualitatively agree with measured data recorded by Fraser-Smith et al. in the immediate hours prior to the earthquake [Fraser-Smith et al., 1990; A. C. Fraser-Smith, personal communication, 2004]. There are remaining uncertainties in this comparison due to inexact knowledge of the lithosphere conductivity and possible instrumentation artifacts acknowledged by Fraser-Smith et al. [1990].

[18] Our ongoing work in this area includes developing an alternative hexagonal/pentagonal 3-D geodesic FDTD grid with superior computational efficiency [Simpson and Taflove, 2004b; Simpson et al., 2005]. We are also planning to couple our EM models to emerging whole-Earth geophysical codes now under development for the study of seismic phenomena, including potential earthquake precursors (APEC Cooperation for Earthquake Simulation
(ACES), available at http://www.quakes.uq.edu.au/ACES/). Our ultimate goal is to provide as rigorous as possible a physics basis for proposed earthquake prediction schemes employing either land-based or satellite-based detectors of ULF/ELF anomalies.

[19] Acknowledgment. This work was partially supported by National Computational Science Alliance under grant number DMS040006N and utilized the Dell Xeon Linux Cluster.

\section{References}

Academic Technologies (2002), MATLAB: The language of technical computing, version 6.5.0.180913a, release 13, Northwestern Univ., Evanston, Ill.

Bannister, P. (1984), ELF propagation update, IEEE J. Oceanic Eng., 9(3), $179-188$.

Bannister, P. (1985), The determination of representative ionospheric conductivity parameters for ELF propagation in the Earth-ionosphere waveguide, Radio Sci., 20(4), 977-984.

Fenoglio, M. A., M. J. S. Johnston, and J. D. Byerlee (1995), Magnetic and electric fields associated with changes in high pore pressure in fault zones: Application to the Loma Prieta ULF emissions, J. Geophys. Res., 100, 12,951-12,958.

Fraser-Smith, A. C., A. Bernardi, P. R. McGill, M. E. Ladd, R. A. Helliwell, and O. G. Villard Jr. (1990), Low-frequency magnetic field measurements near the epicenter of the Ms 7.1 Loma Prieta earthquake, Geophys. Res. Lett., 17, 1465-1468.

Hermance, J. (1995), Electrical conductivity of the crust and mantle, in Global Earth Physics: A Handbook of Physical Constants, AGU Ref. Shelf, vol. 1, edited by T. J. Ahrens, pp. 190-205, AGU, Washington, D. C.

Johnston, M. J. S. (1997), Review of electric and magnetic fields accompanying seismic and volcanic activity, Surv. Geophys., 18, 441-475.

Kulchitsky, A. V., Y. Ando, and M. Hayakawa (2004), Numerical analysis on the propagation of ULF/ELF signals in the lithosphere with highly conductive layers, Phys. Chem. Earth, 29, 495-500.

Majaeva, O., Y. Fujinawa, and M. E. Zhitomirsky (1997), Modeling of non-stationary electrokinetic effect in a conductive crust, J. Geomagn. Geoelectr., 49, 1317-1326.

Molchanov, O. A., M. Hayakawa, and V. A. Rafalsky (1995), Penetration characteristics of electromagnetic emissions from an underground seismic source into the atmosphere, ionosphere, and magnetosphere, J. Geophys. Res., 100, 1691-1712.

Park, S. K., M. J. S. Johnston, T. R. Madden, F. D. Morgan, and H. F. Morrison (1993), Electromagnetic precursors to earthquakes in the ULF band: A review of observations and mechanisms, Rev. Geophys., 31, $117-132$.

Simpson, J., and A. Taflove (2002a), Two-dimensional FDTD modeling of impulsive ELF antipodal propagation about the Earth-sphere, Proc. IEEE Antennas Propag. Soc. Int. Symp., 3, 678-681.

Simpson, J., and A. Taflove (2002b), Two-dimensional FDTD model of antipodal ELF propagation and Schumann resonance of the Earth, IEEE Antennas Wireless Propag. Lett., 1, 53-56.

Simpson, J., and A. Taflove (2003), Global three-dimensional FDTD modeling of impulsive ELF propagation about the Earth, Proc. IEEE Antennas Propag. Soc. Int. Symp., 4, 940-944.

Simpson, J., and A. Taflove (2004a), Three-dimensional FDTD modeling of impulsive ELF propagation about the Earth-sphere, IEEE Trans. Antennas Propag., 52, 443-451.

Simpson, J., and A. Taflove (2004b), Efficient modeling of impulsive ELF antipodal propagation about the Earth sphere using an optimized twodimensional geodesic FDTD grid, IEEE Antennas Wireless Propag. Lett., 3, 215-218.

Simpson, J. J., R. P. Heikes, and A. Taflove (2005), 3-D FDTD modeling of ULF/ELF propagation within the global Earth-ionosphere cavity using an optimized geodesic grid, Proc. IEEE Antennas Propag. Soc. Int. Symp., in press.

Taflove, A., and S. C. Hagness (2000), Computational Electrodynamics: The Finite-Difference Time-Domain Method, 2nd ed., Artech House, Norwood, Mass.

J. J. Simpson and A. Taflove, Department of Electrical and Computer Engineering, Northwestern University, 2145 Sheridan Road, M367, Evanston, IL 60208, USA. (j-simpson@northwestern.edu) 